\title{
Advances in Highly Constrained Multi-Phase Trajectory Generation using the General Pseudospectral Optimization Software $\mathcal{G P O P S}$
}

\author{
Shawn L. Rexius* Tiffany E. Rexius ${ }^{\dagger}$ Timothy R. Jorris ${ }^{\ddagger}$ \\ Edwards AFB, CA, 93524, USA \\ Anil V. Rao ${ }^{\S}$ \\ University of Florida, Gainesville, FL, 32611, USA
}

\begin{abstract}
Recent events in hypersonic glide vehicle programs have necessitated a new approach to examine possible flight test trajectories to include new constraints on optimal trajectory generation. Aircraft stability, combined thermo-structural loading, and vehicle ablation are all important considerations for hypersonic vehicle flight; optimal trajectory generation should account for these complex constraints. A collaborative effort was undertaken by the Air Force Research Laboratory Aerospace Systems Directorate and the $412^{\text {th }}$ Test Wing at Edwards Air Force Base to analyze possible optimal control solutions that satisfy these constraints. A three-stage booster aerodynamic and propulsion model and a hypersonic glide vehicle aerodynamic and ablation model were implemented in the General Pseudospectral Optimization Software $(\mathcal{G P O P S})$. The resulting optimal control problem models booster launch through re-entry vehicle impact and incorporates multiple complex constraints including stagnation heating, ablation, no-fly zones, aircraft stability, dynamic pressure, time-rate of change of flight path angle, loads and a terminal phase target. Furthermore, the optimal control problem uses derived events as guidance mode or booster stage linkages; including range to target, range from launch site, vehicle loads, altitudes, dynamic pressure and time rate of change of altitude. The optimal control variables are specified to be the guidance variable derivatives; this allows for constraining attitude rates in a translational (point mass) problem. $\mathcal{G P O P S}$ is shown to be capable of analyzing highly constrained, multiple-phase optimal control problems using complex vehicle models and flexible enough to incorporate new constraints quickly allowing for exploration of new guidance methodologies.
\end{abstract}

\section{Nomenclature}

A axial force

$a_{x}$ axial acceleration

$a_{z} \quad$ normal acceleration

$C_{A}$ axial force coefficient

$C_{l \beta} \quad$ rolling moment coefficient due to sideslip

$C_{l \delta}$ rolling moment coefficient due to aileron

$C_{N}$ normal force coefficient

$C_{n \beta}$ yawing moment coefficient due to sideslip

$C_{n \delta}$ yawning moment coefficient due to aileron

\footnotetext{
*Project Engineer, Hypersonic Combined Test Force, shawn.rexius.2@us.af.mil, AIAA Member.

${ }^{\dagger}$ Research Analyst, AFRL Aerospace Systems Directorate, tiffany.rexius.1@us.af.mil, AIAA Member.

¥Director, Hypersonic Combined Test Force, timothy.jorris@us.af.mil, AIAA Member.

§Professor, Department of Mechanical and Aerospace Engineering, anilvrao@ufl.edu, AIAA Member.

The views expressed are those of the author and do not reflect the official policy or position of the Department of Defense or the U.S. Government. Distribution A: Approved for public release; distribution unlimited. PA Number 412-TW-PA-13395
} 


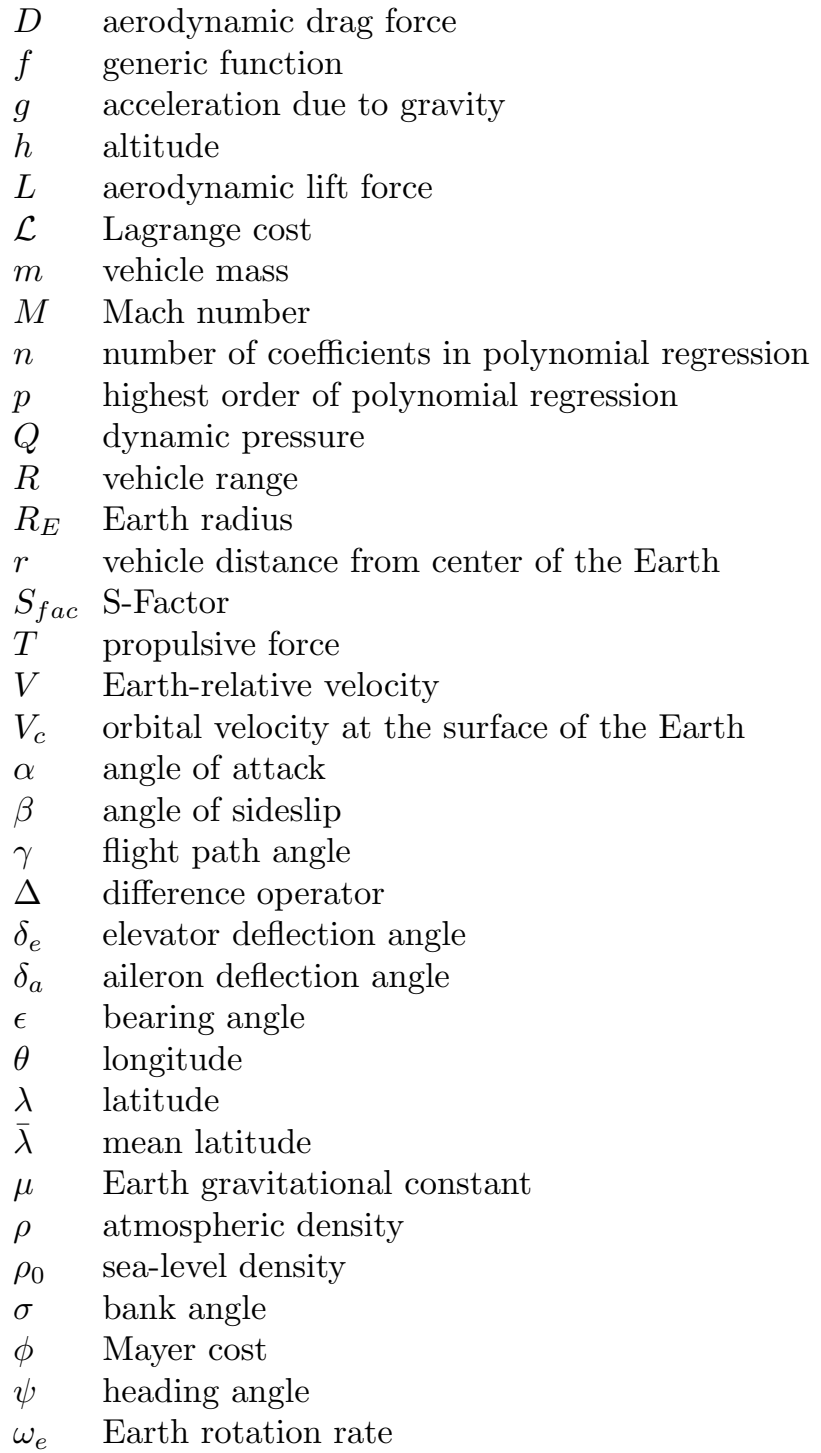

\section{Introduction}

Recent events in hypersonic glide vehicle flight testing have proven hypersonic gliding flight to be a highly constrained problem. Combined aerodynamic and thermodynamic loading, advanced material uncertainties, vehicle stability and control, and thermal protection system constraints have become important considerations when designing trajectories for long range hypersonic glide. Furthermore, geo-political implications and range safety concerns may dictate no-fly zones as additional constraints. These classes of problems are suitable for pseudospectral optimal control because of the highly constrained solution space and the need to assess the effectiveness of various guidance methodologies; also, pseudospectral methods are useful for multiple-phase problems.

Previously, it was shown that pseudospectral optimal control was suitable for Common Aero Vehicle $(\mathrm{CAV})$ re-entry trajectories constrained by waypoints and no-fly zones. ${ }^{1-3}$ This work was then expanded from the purely analytical cases to model a CAV in flight about a spherical rotating Earth. ${ }^{4}$ Further expansion of the CAV re-entry problem added aerodynamic heating path constraints. ${ }^{5}$ Lately, pseudospectral methods have shown their versatility for hypersonic flight testing by finding optimal control solutions considering range safety implications. ${ }^{6}$

Common to the above work is the use of the General Pseudospectral Optimization Software $(\mathcal{G P O P S})$ analysis progam ${ }^{7-11}$ that has been adapted by the $412^{\text {th }}$ Test Wing and the Air Force Research Laboratory 
at Edwards AFB specifically for solving optimal control problems involving hypersonic and space-access vehicles. $\mathcal{G P O P S}$ is a MATLAB-based hp-adaptive pseudospectral optimization software. $\mathcal{G P O} \mathcal{P} \mathcal{S}$ utilizes a Radau Pseudospectral Method (RPM); the collocation points are defined by the roots of Legendre-GaussRadau (LGR) functions. ${ }^{9} \mathcal{G} \mathcal{P O P S}$ also automatically refines the "mesh" by subdividing time segments of the state and control solution to arrive at a denser solution without using higher-order polynomials and their concerns; this is called an $h p$-adaptive mesh. $\mathcal{G P O P S}$ ' MATLAB implementation allows the user to include any number of new constraints, events, links, costs and even equations of motion and take advantage of the ease of debugging and matrix operations within MATLAB.

The work presented for this effort is the implementation of multiple complex models representing a threestage booster and a hypersonic glide vehicle (HGV) similar to the CAV within the $\mathcal{G P O P S}$ framework. The resulting optimal control problem has variable events for stage and fairing separation, payload separation, and re-entry guidance mode phases. Furthermore, complex constraints are imposed on the booster and reentry problems; to include aerodynamic, aero-thermodynamic, aircraft stability, vehicle loads and multiple no-fly zones. Finally, various guidance methodologies are explored for use with the HGV. It is shown that $\mathcal{G P O P S}$ can produce optimal control solutions for these types of problems.

\section{Methodology}

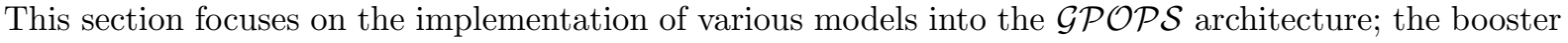
model, the HGV model, the various Earth models and equations of motion are discussed. Additionally, the limits, constraints, phases, and cost function for the optimal control problem are discussed.

\section{II.A. Booster Model}

A three stage launch vehicle with solid rocket motors was modeled for the booster as seen in Figure 1. The

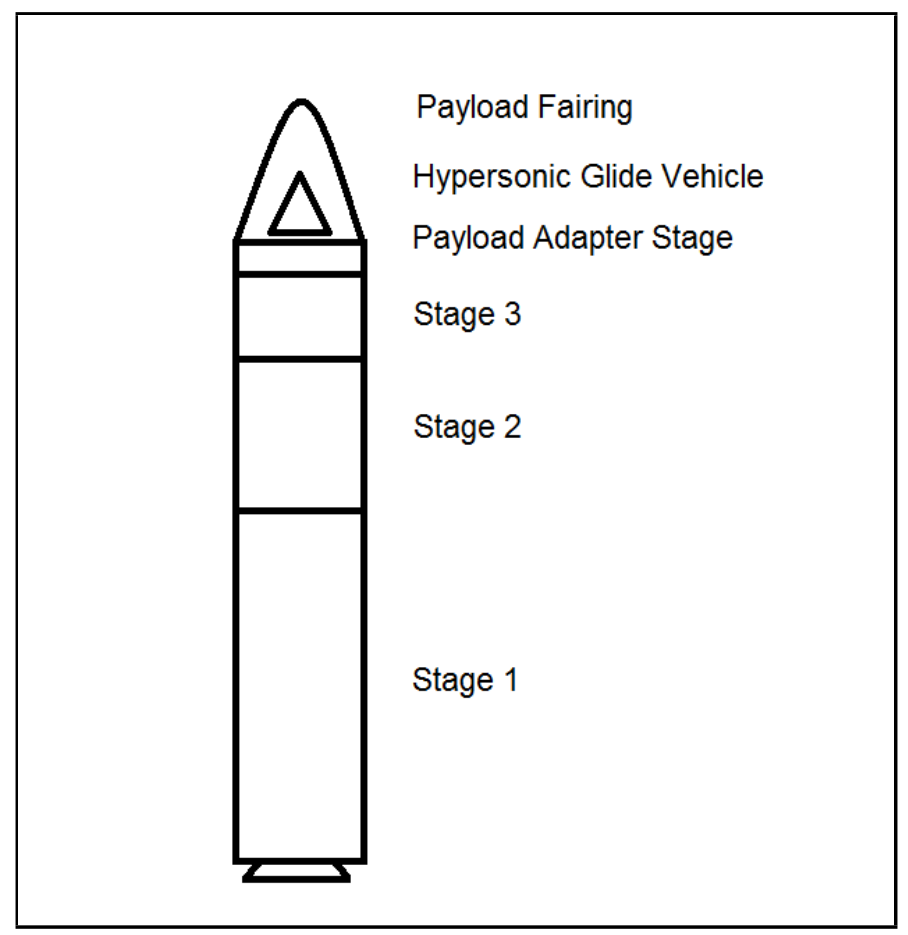

Figure 1. Booster Stack with HGV.

booster consisted of three stages with a fairing separation occurring during the third stage. After fairing separation the vehicle coasted until the correct conditions were met and the HGV separated. Thrust and mass flow rate for all stages were from table look-ups based on time; thrust was adjusted for altitude utilizing a custom atmosphere. Throttling was applied to the third stage of the booster at a specified time. The aerodynamic model is a two-dimensional table look-up for axial force coefficent as a function of Mach number 
and angle of attack and changes with each vehicle stage, Eq (1):

$$
C_{A}=f(M, \alpha)
$$

For this booster model the normal force coefficient $\left(C_{N}\right)$ was assumed to be zero. The normal force to the booster is generally small enough for this to be a valid assumption. The controls to be optimized were the angle of attack, sideslip, and bank angle. Rate limits were applied to these controls to ensure feasible solutions based on vehicle maneuvering capability. Significantly, using this method agreed with higher degrees of freedom simulations.

The booster model was broken into four separate phases due to discontinuities in mass. The dry mass of the stage was ejected after stage burnout resulting in a discontinuity in the state of mass necessitating a phase break. This happened between each phase as well as for fairing separation which was only able to occur above a certain altitude and below a specified acceleration.

\section{II.B. Hypersonic Glide Vehicle Model}

The payload for the booster was modeled as a HGV similar to the CAV. ${ }^{1-5}$ However, the CAV model presented in previous work utilized a simple drag polar with a constant lift coefficient that approximates Mach-independent maximum glide range flight. ${ }^{3}$ The aerodynamic model in this work is a more representative vehicle model originally used for explicit simulation as a four-dimensional table-look-up as a function of Mach number $(M)$, altitude $(h)$, angle of attack $(\alpha)$, and elevator deflection $\left(\delta_{e}\right)$ Eq (2):

$$
C_{A}, C_{N}=f\left(M, h, \alpha, \delta_{e}\right)
$$

Furthermore, each force coefficient has two sets of tables for laminar and turbulent boundary layers. Boundary layer transition was based on Reynolds number and angle of attack and linearly faired between laminar and turbulent. For computational speed and ability to utilize GPOPS' automatic differentiation option, the laminar and turbulent four-dimensional tables were fit to polynomials at an approximate trim elevator deflection, $\mathrm{Eq}(3)$ :

$$
C_{A}, C_{N}=C_{0}+C_{1} M+C_{2} h+C_{3} \alpha+C_{4} M^{2}+C_{5} h^{2}+C_{6} \alpha^{2}+\ldots C_{n-2} M^{p}+C_{n-1} h^{p}+C_{n} \alpha^{p}
$$

The polynomial fit was accomplished by fitting a "response surface" or regression to the tabular data using a method similar to Waddell. ${ }^{12}$ It is important to note that in some cases multiple surfaces were fit to the tabular data for a single table to avoid higher-order interpolation errors. In those cases the multiple surfaces were fit together using a linear fairing.

An important consideration for hypersonic glide vehicles is aircraft stability and control. A measure of performance of aircraft lateral-directional stability and control is the Lateral Control Departure Parameter (LCDP) $;^{13}$ this is similar to the S-Factor $\left(S_{f a c}\right)$ parameter used in this work, Eq (4):

$$
S_{f a c}=1-\frac{C_{l \beta} C_{n \delta}}{C_{n \beta} C_{l \delta}}
$$

The above S-Factor calculation is applicable for aircraft that have no yaw control; it is an indicator of departure resistance with values greater than zero. ${ }^{14}$ Instead of modeling four variables on the right hand side of $\mathrm{Eq}$ (4) the composite value for $S_{f a c}$, the left hand side of $\mathrm{Eq}$ (4), was modeled. To that end, a response surface for S-Factor - the left hand side of Eq (4) - was fit for laminar and turbulent tabular models. In addition to elevator deflection, derivatives due to aileron were also a function of aileron deflection. Aileron deflection was chosen conservatively and held as a constant for the fit in addition to the same constant elevator deflection as in the axial and normal force coefficients. In this way a lateral-directional stability constraint was added to the aircraft model without modeling the lateral-directional dynamics.

Another important consideration for re-entry vehicles is ablation or recession; the trajectory flown is constrained by ablation limits. In this study nosetip ablation was considered; an empirical equation is used to calculate the rate-of-change of nosetip ablation as a function of atmospheric density $(\rho)$ and velocity $(V)$, Eq (5):

$$
\dot{R}_{a b l}=B \rho^{k_{1}} V^{k_{2}}
$$

Note that wing leading edge recession will generally be of greater importance than nosetip; for this study the nosetip recession is used in a conservative fashion to stand for recession in general. More detailed studies must consider more than nosetip recession for long range hypersonic glide. 


\section{II.C. Equations of Motion and Earth models}

The equations of motion are for translational flight about a rotating spherical earth. These equations are derived in $\operatorname{Vinh}^{15}$ and have been modified to include sideslip angle, Eqs (6-11):

$$
\begin{aligned}
\dot{r}= & V \sin \gamma \\
\dot{\theta}= & \frac{V \cos \gamma \sin \psi}{r \cos \lambda} \\
\dot{\lambda}= & \frac{V \cos \gamma \cos \psi}{r} \\
\dot{V}= & \frac{1}{m}(T \cos \alpha \cos \beta-D \cos \beta)-g \sin \gamma+\omega_{E}^{2} r \cos \lambda(\sin \gamma \cos \lambda-\cos \gamma \cos \psi \sin \lambda) \\
\dot{\gamma}= & \frac{T}{m V}(\cos \alpha \sin \beta \sin \sigma+\sin \alpha \cos \sigma)+\frac{1}{m V}(L \cos \sigma-D \sin \sigma \sin \beta)+\frac{V}{r} \cos \gamma-\frac{g}{V} \cos \gamma \\
& +2 \omega_{E} \sin \psi \cos \lambda+\frac{\omega_{E}^{2}}{V} r \cos \lambda(\cos \gamma \cos \lambda+\sin \gamma \cos \psi \sin \lambda) \\
\dot{\psi}= & \frac{-T}{m V \cos \gamma}(\cos \alpha \sin \beta \cos \sigma+\sin \alpha \sin \sigma)-\frac{1}{m V \cos \gamma}(L \sin \sigma-D \cos \sigma \sin \beta)+\frac{V}{r} \cos \gamma \sin \psi \tan \lambda \\
& -2 \omega_{E}(\cos \lambda \tan \gamma \cos \psi-\sin \lambda)+\frac{\omega_{E}^{2}}{V \cos \gamma} r \sin \lambda \cos \lambda \sin \psi
\end{aligned}
$$

In Equations (6-11) above, the sideslip angle $(\beta)$ and thrust $(T)$ were assumed to be zero for the HGV portion of the optimal control problem; however, the booster uses sideslip in addition to angle of attack $(\alpha)$ and bank angle $(\sigma)$. The Earth's gravitational field was modeled by the following equation, Eq. (12):

$$
g=\frac{\mu m}{r^{2}}
$$

The Earth's atmosphere was modeled with a 1962 standard atmosphere ${ }^{16}$ for the HGV and a custom atmosphere table for the booster; this was used to be consistent with the aforementioned explicit simulation.

\section{II.D. Phases, Constraints and Limits}

The optimal control problem was subdivided into multiple phases to account for booster stages, payload fairing separation, payload separation, and re-entry guidance phases. The booster modeled was a three-stage launch vehicle with a payload fairing and a fourth stage with no propulsion that separates the hypersonic glide vehicle shown in Figure 1.

Optimal control problems in $\mathcal{G P O P S}$ are constrained by the equations of motion and user-defined constraints enforced throughout the phase called path constraints. Furthermore, $\mathcal{G P O P S}$ problem phases can have user-defined constraints at the start and end points of the phase called events; user-defined constraints that exist between phases are called linkages.

The booster has three controls, angle of attack, bank angle, and sideslip angle. In order to more closely approximate vehicle dynamics, the controls for the optimal control problem are the angle of attack, bank angle, and sideslip angle derivatives. In this way, the rate of change of control can be constrained. The boost phases are shown in Figure 2. 


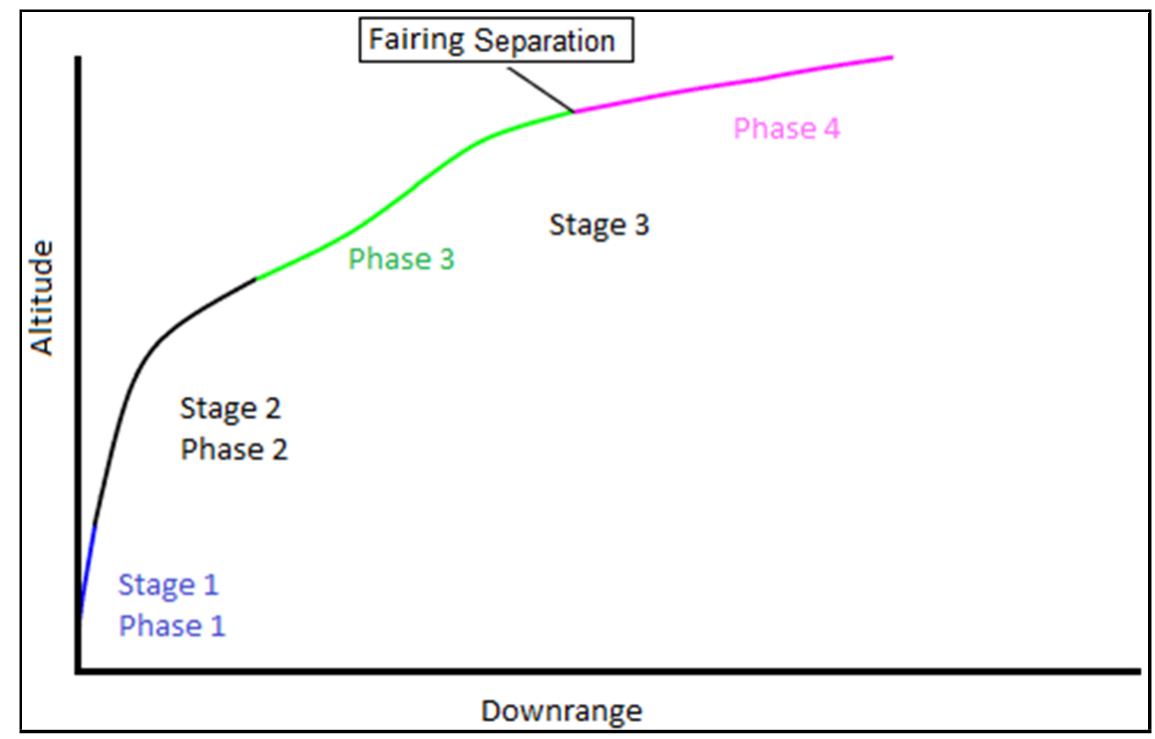

Figure 2. Booster Optimal Control Problem Phases.

Booster phase 1 starts 5 seconds after liftoff to avoid numerical difficulties in the equations of motion at zero velocity and ends at stage 1 burnout; as the booster vehicle dynamics are well understood this is a valid starting point. In booster phase 1 , there is a path constraint of "Q-Alpha" $(Q \cdot \alpha)$, a measure of aerodynamic loading that leads to bending of the launch vehicle. Q-Alpha is defined by the following equation, Eq (13):

$$
Q \cdot \alpha=\frac{\alpha \rho V^{2}}{2}
$$

In the above equation, angle of attack $(\alpha)$ is in degrees and dynamic pressure $\left(1 / 2 \rho V^{2}\right)$ is in units of pounds per square foot. Booster phase 1 also has an event constraint; the stage separation must occur within a certain range of Q-Alpha. The stage separations were modeled as an instantaneous mass change with no dynamic effects on the launch vehicle.

Booster phase 2 starts at stage 2 ignition and ends at stage 2 separation; phase 2 also has a path constraint of Q-Alpha. Booster phase 3 starts at stage 3 ignition and ends at fairing separation; there are no path constraints in phase 3 . The stage separation event must occur within a certain range of axial acceleration and altitude. Axial acceleration for the vehicle is defined by the following equation as a function of booster thrust $(T)$, axial force $(A)$ and mass $(m), \mathrm{Eq}(14)$ :

$$
a_{x}=\frac{T-A}{m}
$$

Booster phase 4 starts at fairing separation and ends at HGV separation; there are no path constraints in phase 4. It is assumed that the time between fairing separation and HGV separation must be greater than a minimum time for navigation subsystem acquisition. Stage 3 separation must occur after the thrust tail-off reaches a certain point; therefore the event constraint in phase 4 is the stage 3 thrust level. Glide vehicle separation must occur within a certain altitude and flight path angle range.

The HGV has two controls, angle of attack and bank angle. As with the booster, the controls specified in the optimal control problem are the angle of attack and bank angle derivatives. There are six glide phases from booster separation to impact shown in Figure 3. 


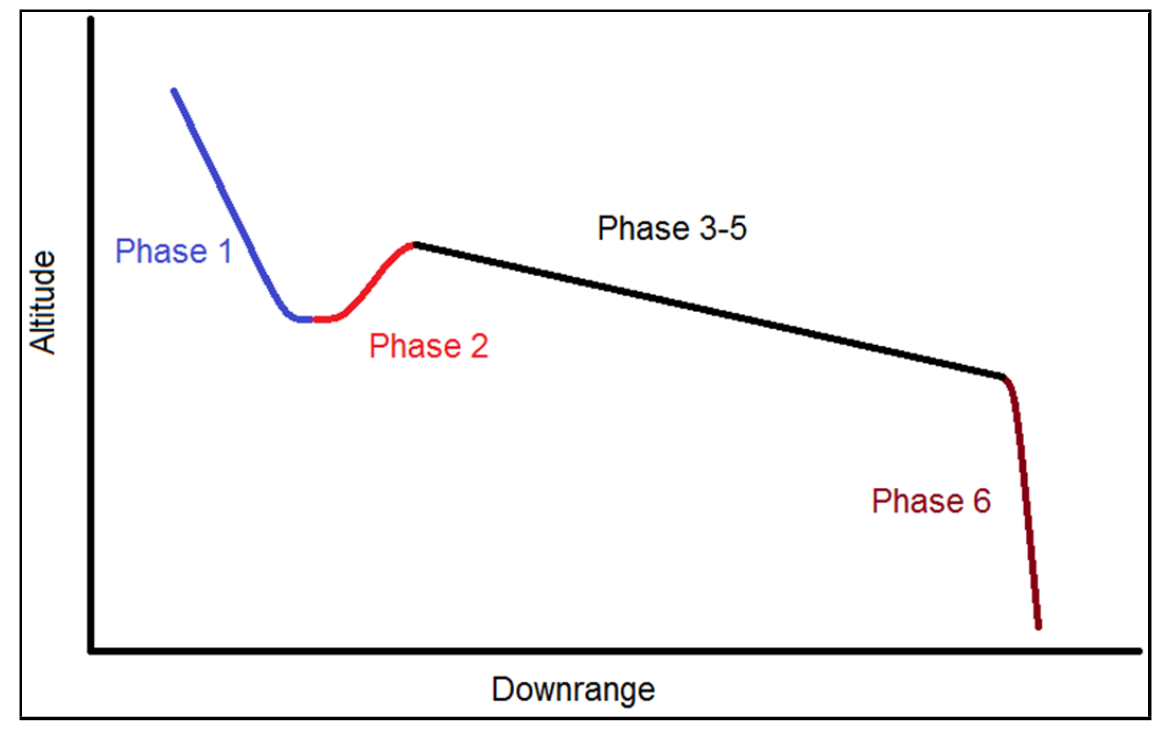

Figure 3. HGV Optimal Control Problem Phases.

Throughout the entire HGV trajectory, $S_{f a c}$ is applied as a path constraint; as mentioned in the previous section $S_{f a c}$ must be above zero for the vehicle to avoid departure of controlled flight. Cumulative vehicle nose recession is applied as a constraint to the entire trajectory.

HGV phase 1 starts at glide vehicle separation; this phase is called the exo-atmospheric coast and ends when there is sufficient lift on the glide vehicle to zero the flight path angle. The separation point (initial phase point) must be within a certain range from the launch site and the final phase point must have a flight path angle of zero.

HGV phase 2 starts at the end of phase 1 and ends once the HGV has entered the proper pseudoequilibrium glide condition. The proper pseudo-equilibrium glide is defined for this problem as an altitude and a flight path angle. An event was defined at the end of this phase as the miss distance for the desired altitude; the desired altitude is a parameter in $\mathcal{G P O P S}$ that the optimal control problem selects as part of the optimization.

HGV phases 3 through 5 are glide phases and comprise the majority of the HGV trajectory. The HGV glide has been broken into three separate phases in order to experiment with different options for HGV guidance and a variety of guidance variables and methodologies. The HGV glide phase ends at the interface to the terminal guidance mode switch; this is a range to impact location, altitude, velocity, and flight path angle. The range to impact location is estimated on the spherical earth from the law of cosines, Eq (15):

$$
R=R_{E} \cos ^{-1}\left(\sin \lambda_{1} \sin \lambda_{2}+\cos \lambda_{1} \cos \lambda_{2} \cos \left(\theta_{1}-\theta_{2}\right)\right)
$$

The numbered subscripts in Eq (15) denote the vehicles and the targets position, respectively. To ensure that the HGV was pointing at the impact location at the end of the glide, a final event constraint was used; the HGV heading was constrained to match the HGV bearing to the impact location. The bearing was estimated by the following equation; a local flat-earth approximation corrected for latitude, Eq (16):

$$
\epsilon=\tan ^{-1}\left(\frac{\Delta \lambda}{\Delta \theta}\right)
$$

Because the HGV is relatively close to the impact location at the end of the glide, the local-flat Earth approximation for bearing was decided to be appropriate.

The final HGV phase is the end of the glide to the impact at the impact location. The path constraints in this phase are normal and axial acceleration. The impact must be within a certain flight path angle, velocity and range to the impact location. 


\section{II.E. Cost Function}

The cost, or objective, function in $\mathcal{G P O P S}$ is defined in two parts; an endpoint cost, and an integrated cost, Eq (17):

$$
J=\phi+\int \mathcal{L}
$$

In Eq (17), The endpoint cost $(\phi)$ is called the Mayer cost, and the integrand $(\mathcal{L})$ is called the Lagrange cost.

The cost function for the optimal control problem was chosen to minimize the stagnation heating at the end of HGV phase 1, that is, the maximum heating experienced by the HGV prior to the terminal phase of flight. The stagnation heating rate is given by the Chapman equation, ${ }^{17} \mathrm{Eq}$ (18):

$$
\dot{q}_{s}=\hat{Q}\left(\frac{\rho}{\rho_{0}}\right)^{0.5}\left(\frac{V}{V_{C}}\right)^{3.15}
$$

In Eq (18), the constant $(\hat{Q})$ is a function of the radius of the nose. The above cost function is modeled as a Mayer cost; the Lagrange cost is zero. Note that this cost function is applied to the endpoint of one phases; In $\mathcal{G P O P S}$, multiple endpoint or integral cost functions may be used; but for this work only one was needed.

\section{Results}

The methodology described in the previous section was implemented and verified against the explicit simulations from which the models were taken. Following verification, the $\mathcal{G P O P S}$ combined booster and HGV optimal control problem was used to develop trajectories. The effect of the chosen cost function was examined along with the effects of the constraints discussed in the methodology section. Furthermore, the effectiveness of different glide phase guidance methods were examined.

Recall that the cost function for the optimal control problem is to minimize stagnation heating. The first set of results show the comparison between the original trajectory and the remediated $\mathcal{G P O P S}$ trajectory. Figure 4 shows the difference in boost and exo-atmospheric altitude profiles. In order to minimize the stagnation heating, the optimal solution has significantly changed the boost profile in order to reduce the apogee altitude by approximately 30 percent. Figure 5 displays that in addition to reducing the apogee altitude, the optimal solution increases the booster velocity and lowers the separation flight path angle. Also with regard to the change in booster controls, Figure 6; the optimal solution uses a different combination of angle of attack and sideslip to change the boost profile.

During the exo-atmospheric glide phase, the HGV angle of attack is raised until the stability factor $\left(S_{f a c}\right)$ path constraint is a concern, Figure 7. Recall that the constraint is for the stability factor to remain positive; the more positive, the better. Note that as the stability path constraint becomes a concern to the optimal control problem, the HGV angle of attack is lowered to the extent allowable to satisfy the path constraint. The $S_{f a c}$ path constraint and the cost function are in opposition during the exo-atmospheric glide phase; totally minimizing stagnation heating at perigee would give an optimal control of higher angle of attack which in turn would violate the stability path constraint. The effect of the changes in booster and re-entry profiles is seen in Figure 8; the $\mathcal{G P O P S}$ optimal solution lowers the perigee heat flux rate by approximately 20 percent. 


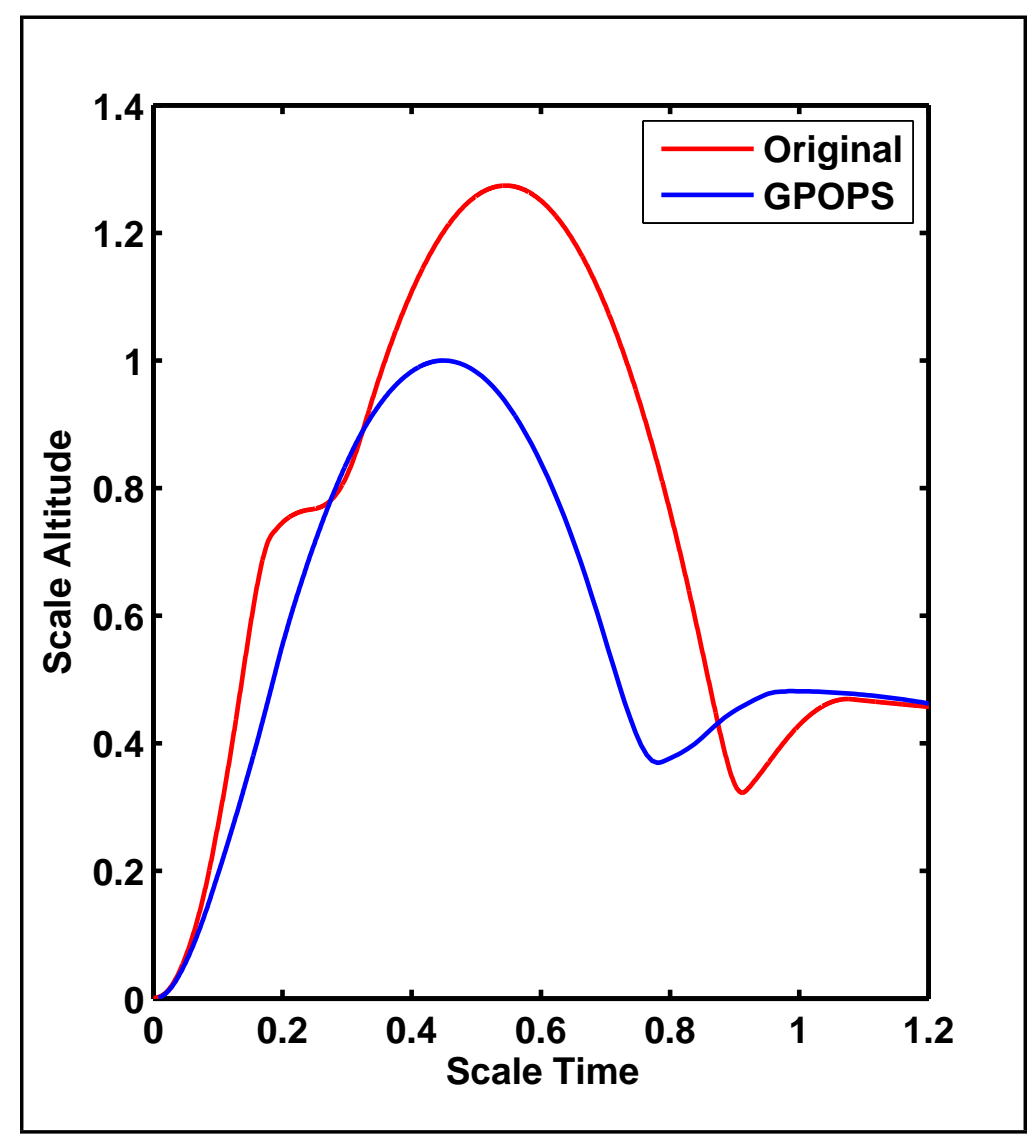

Figure 4. Booster and HGV Re-entry Altitude Profile Comparison.

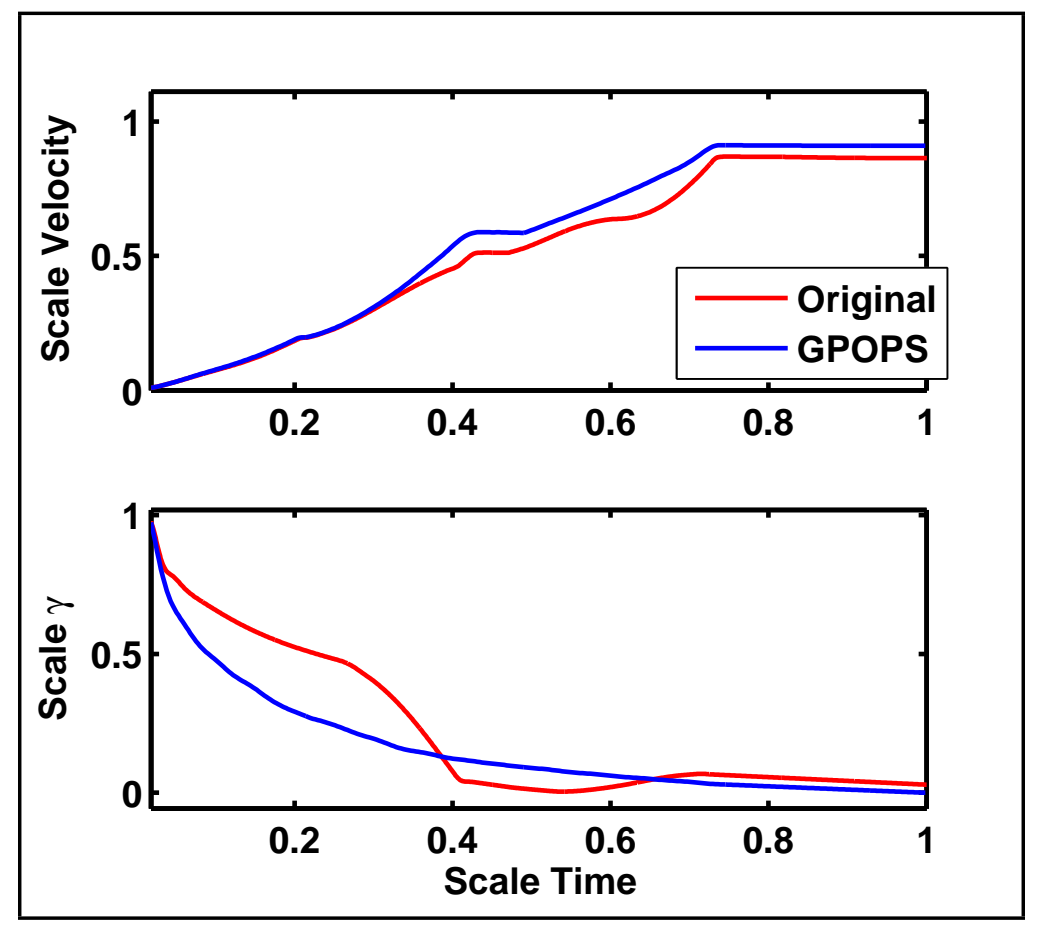

Figure 5. Booster Velocity and Flight Path Angle Comparison. 


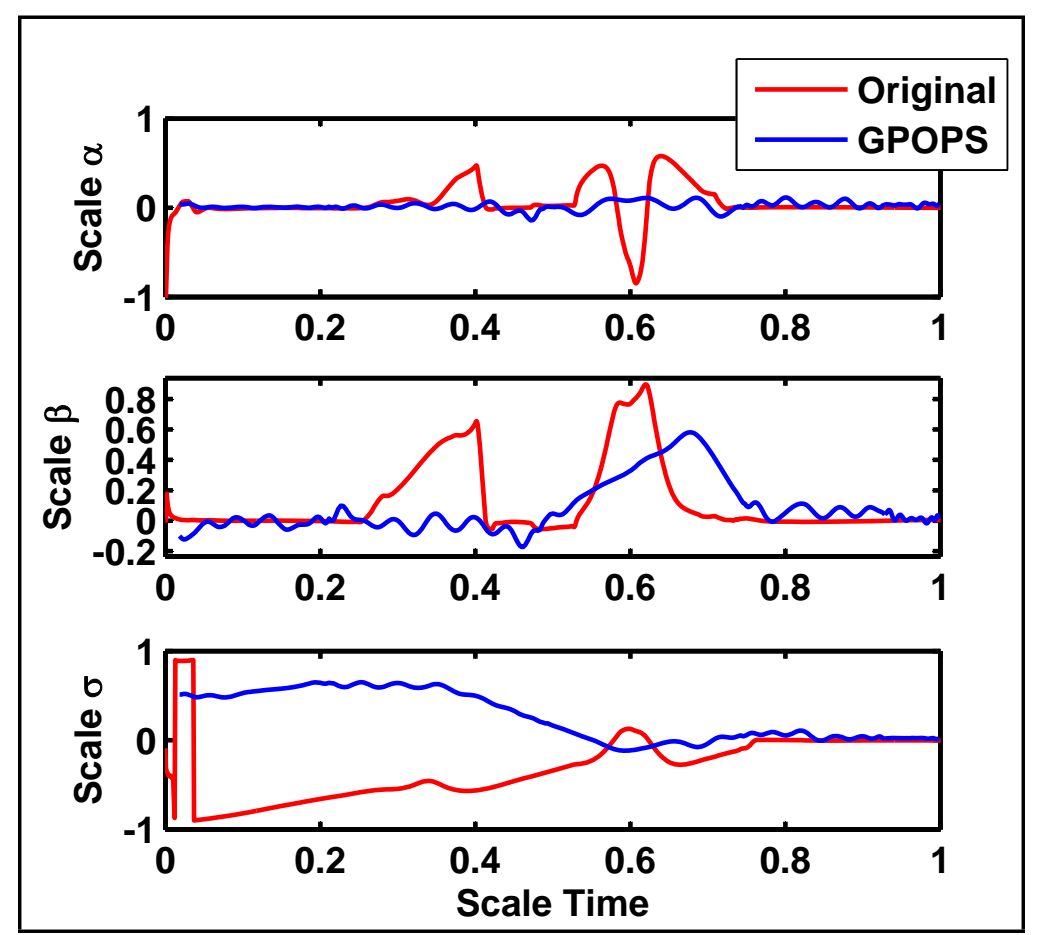

Figure 6. Booster Controls Comparison.

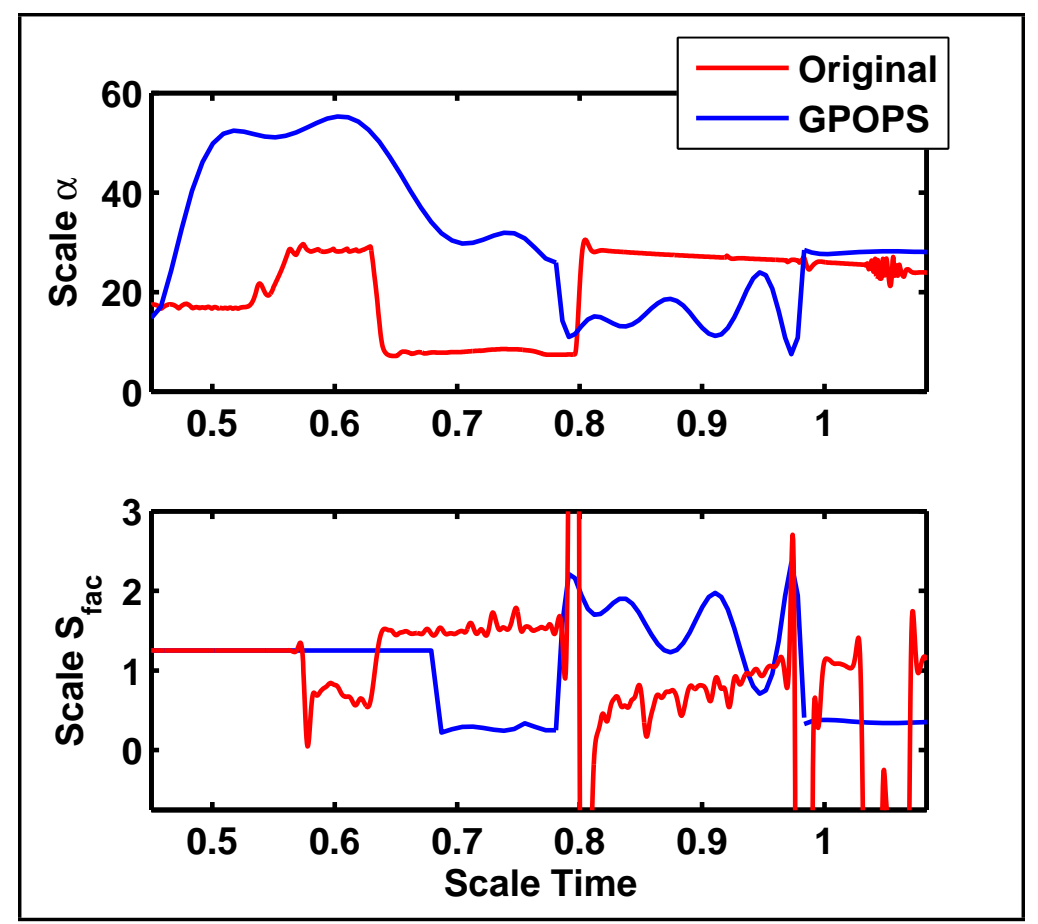

Figure 7. HGV Angle of Attack and Stability Factor Comparison. 


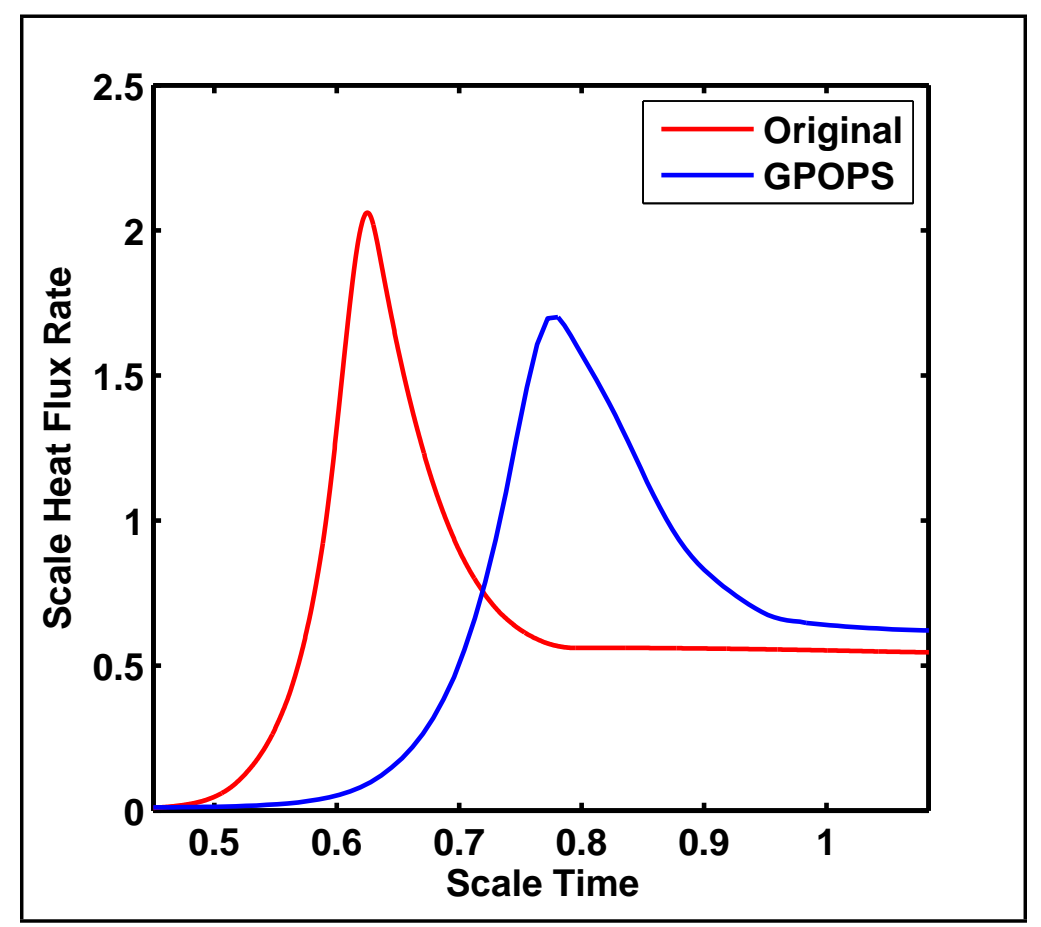

Figure 8. HGV Stagnation Heat Flux Rate Comparison.

In addition to using the $\mathcal{G P O P S}$ booster and HGV optimal control problem to analyze the effect of minimizing stagnation heating at the perigee of the HGV re-entry, no-fly-zones were also used to constrain the flight path of the HGV and booster. The effect of using a no-fly-zone is shown in Figure 9; the unconstrained trajectory traverses through the no-fly-zone on the original optimal solution and the constrained trajectory shifts the ground-track to a new optimal solution satisfying the path constraint.

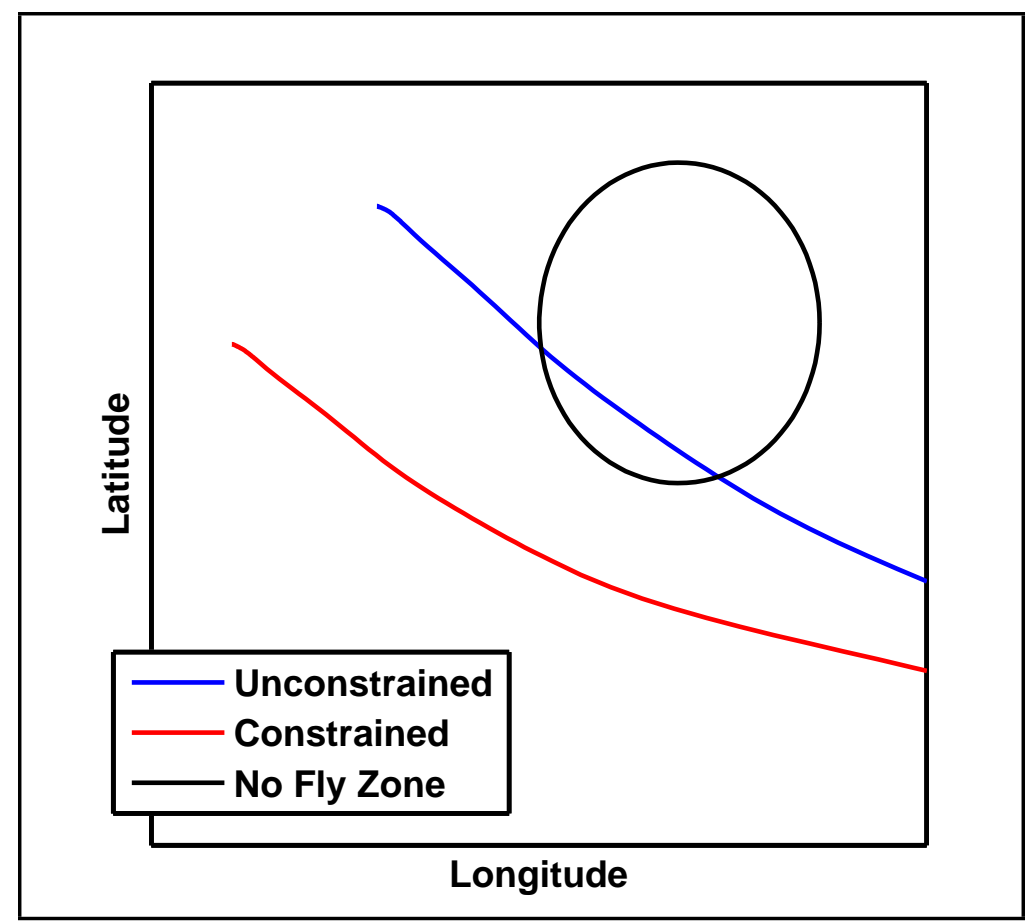

Figure 9. Effect of No-Fly-Zone Path Constraint on HGV Ground-Track.

An endpoint constraint was defined as nose-tip ablation; the allowable nose-tip ablation was constrained 
conservatively for safety margin. Figure 10 shows the effect of the ablation constraint on the re-entry velocity profile; the constrained trajectory essentially flies an entirely different re-entry by reducing the insertion velocity. Note that in Figure 10 the scale time starts at booster ignition but only the re-entry velocity profiles are displayed. The ablation factor profiles are shown in Figure 11. The rate of ablation is initially increased but the final integrated value of ablation is significantly less than the unconstrained trajectory, thus satisfying the new constraint in addition to the previously mentioned constraints.

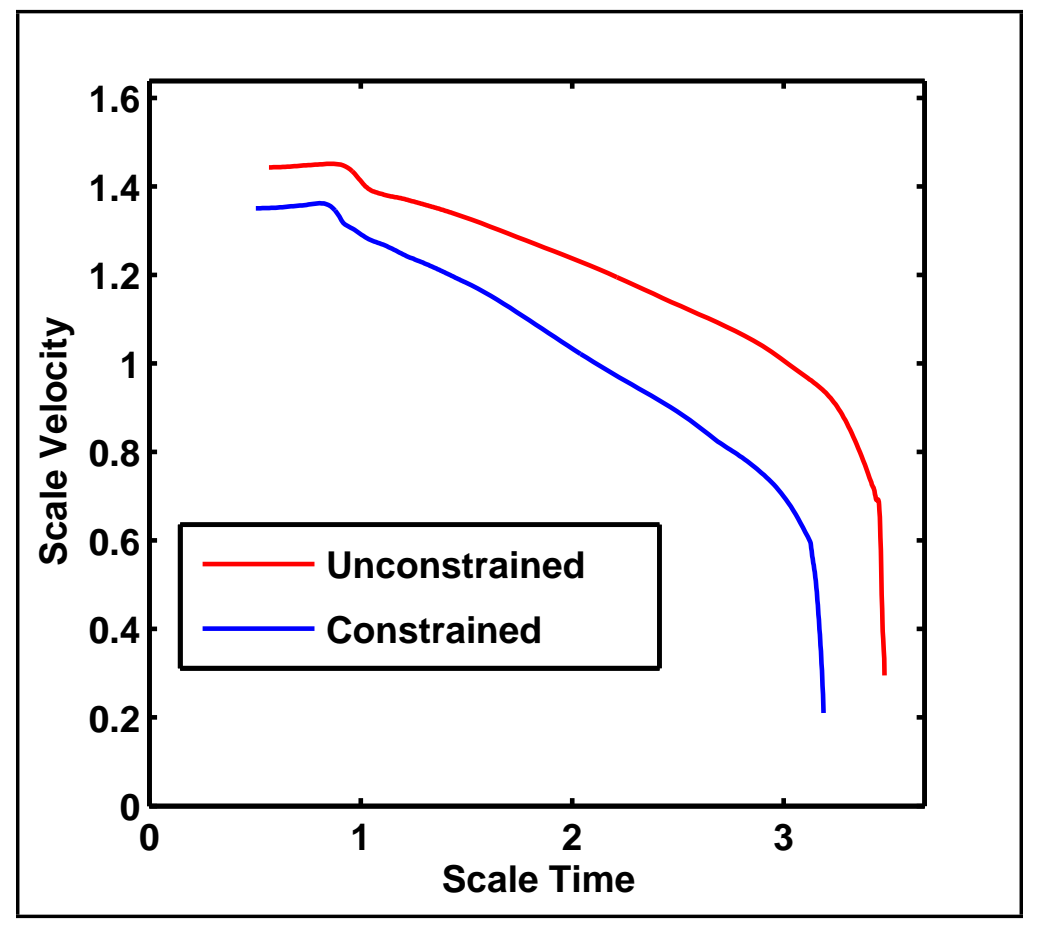

Figure 10. Effect of Ablation Endpoint Constraint on Re-entry Velocity Profile.

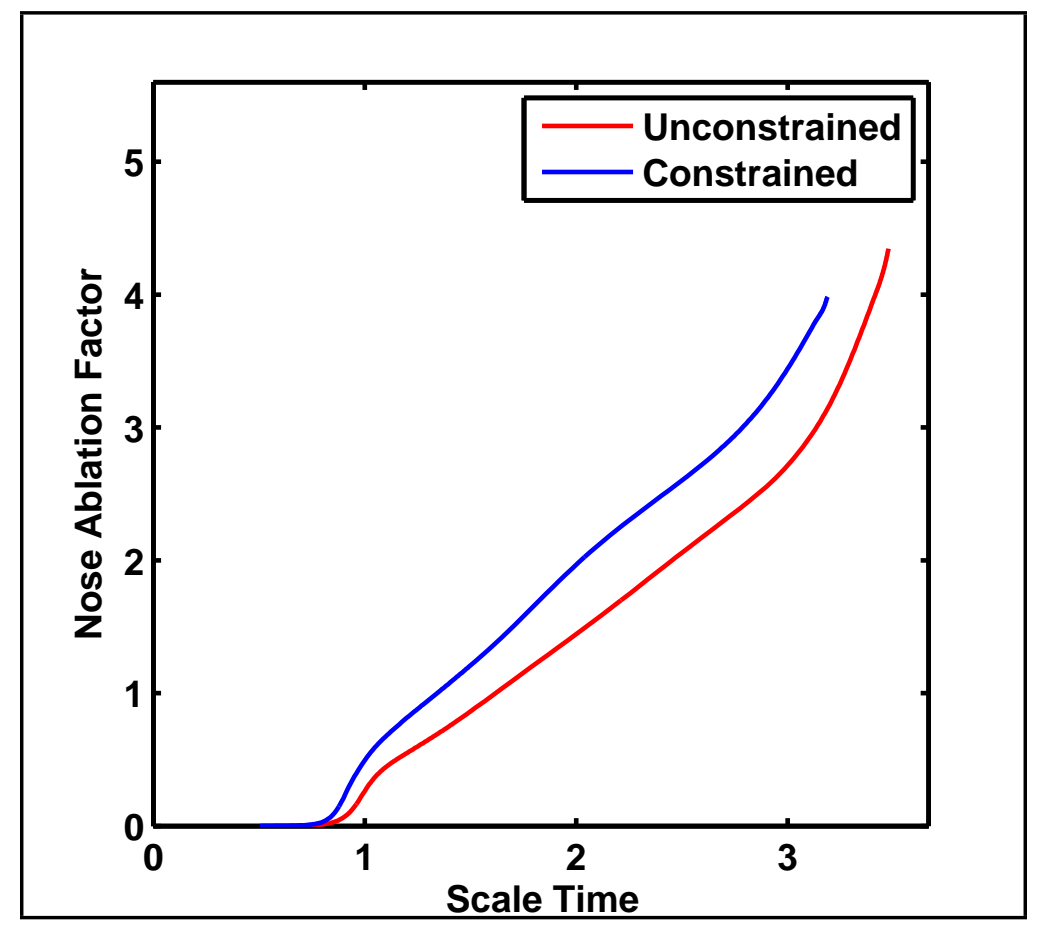

Figure 11. Ablation Factor Comparison. 
More recently, the $\mathcal{G P O P S}$ combined booster and HGV optimal control problem was used to examine the effect of different guidance methodologies in the glide phase with all other constraints still applied; recession, heat rate, stability, etc. The overall altitude profiles of three such methodologies are seen in Figure 12. Guidance 1 uses multiple semi-constant glide slopes during the glide phases. Guidance 2 allows the final glide sub-phase to perform a pop-up maneuver by un-constraining the constant flight path angle in that phase. Guidance 3 allows for more of a phugoid motion by un-constraining the flight path angle during all of the glide phases.

The re-entry angle of attack and flight path angles of the three guidance methodologies are shown in Figure 13. The two options that un-constrain the gliding flight path angle exhibit more movement in angle of attack and more significant longitudinal maneuvering near the terminal phase of the re-entry. Interestingly, the Guidance 1 methodology that enforces a more constant flight path exhibits a steeper initial re-entry.

The aero-thermodynamic effect of the different guidance methodologies is shown in Figure 14; There is a consequence for each guidance methodology. The Guidance 1 option has higher stagnation heating at the perigee but accumulates less heat flux overall; this is favorable for nosetip recession. The Guidance 2 option may be the worst option for aero-thermodynamic constraints as it has higher heating at the perigee and the terminal phase. The Guidance 3 option has the least heating at the perigee and a slower growth of nose ablation but a larger integral heating and a spike of heat flux at the terminal phase. Of the three options with respect to aero-thermodynamic factors, the Guidance 3 option may be the most acceptable overall; the perigee heat flux rate is very important for hypersonic glide vehicles. The aero-thermodynamic consequences of each guidance methodology is shown as an example; the selection of a particular guidance methodology will certainly depend upon many factors. For a re-entry vehicle such as an HGV, to first order the most important considerations have to do with aerodynamic heating.

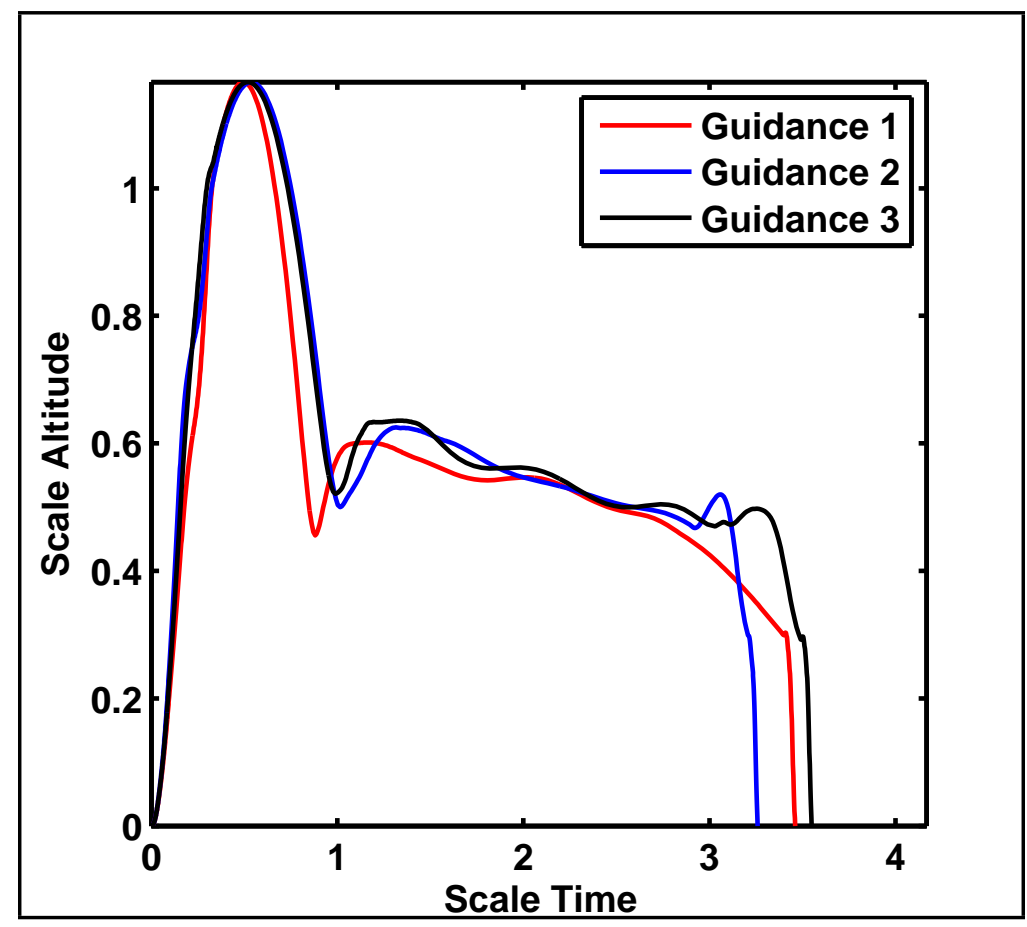

Figure 12. Altitude Profiles of Guidance Methodolgy Options. 


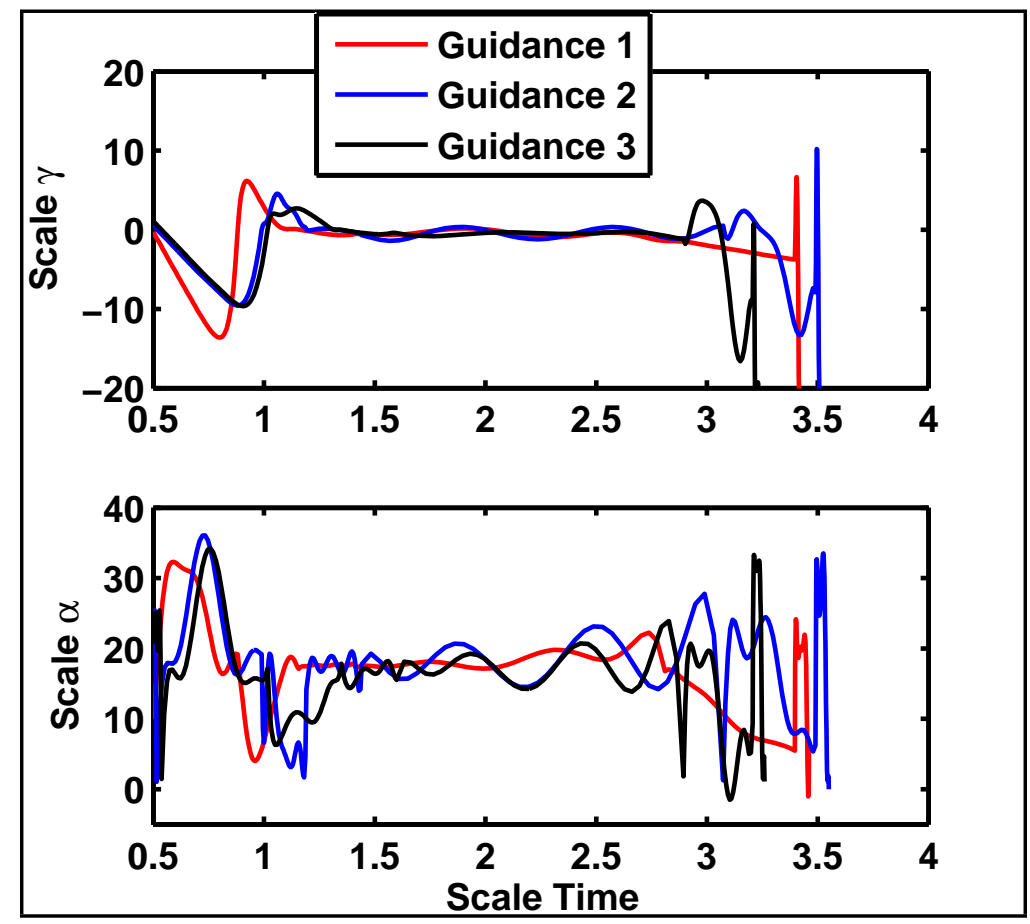

Figure 13. Angle of Attack and Flight Path Angle Profiles for Guidance Methodologies.

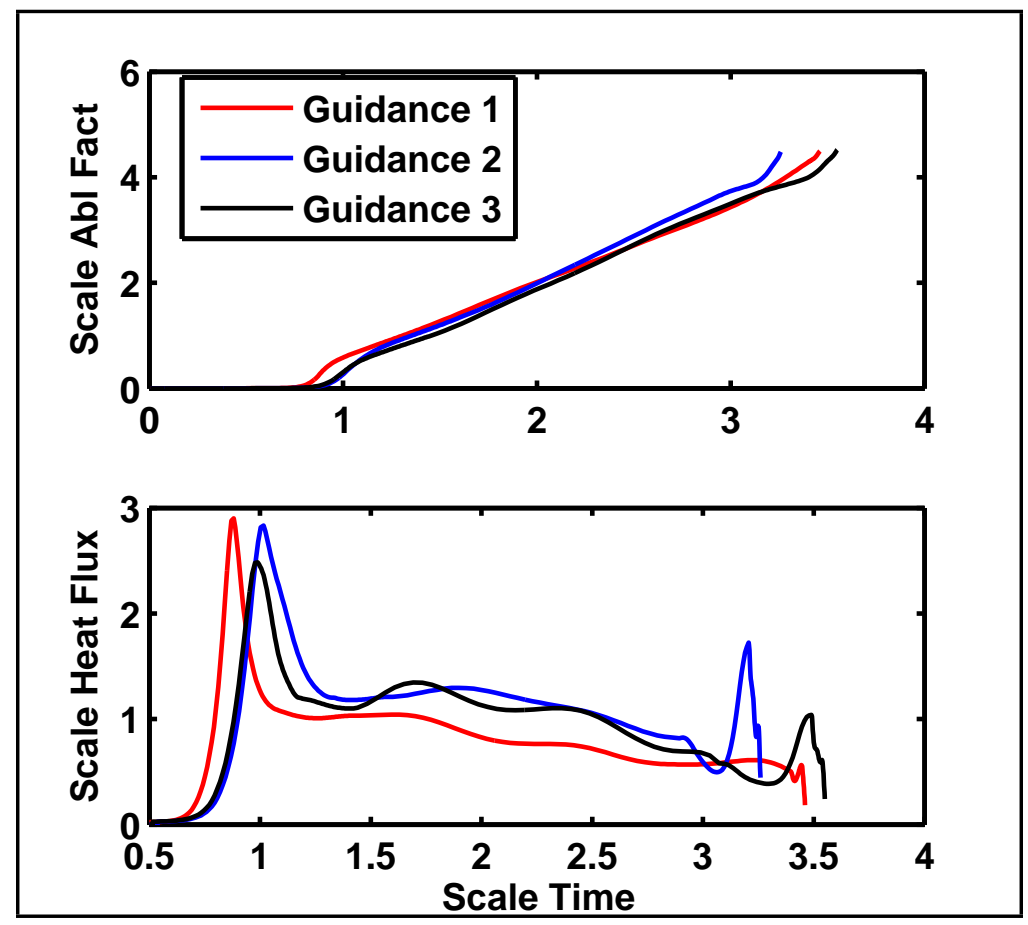

Figure 14. Ablation Factor and Heat Flux Rate Comparison for Guidance Methodologies.

A few challenges in producing results are worth discussion; the implementation of this type of problem revealed many aspects of optimal control problems for further study. First, implementing complex aerodynamic or other model tables can result in models that do not necessarily have smooth derivatives; when the derivatives are not smooth, the solver has a more difficult time finding an optimal solution. This was evident in the booster dynamics since they had no regression fits.

Secondly, $\mathcal{G P O P S}$ method for auto-scaling the variables and constraints was found to be very sensitive 
to small changes in the state, path, and endpoint constraint limits; the difference between a fully optimized solution within the specified tolerances and a solution that did not solve satisfactorily was found to be very small in some cases. For complex problems like the one considered in this work, a manual scaling scheme may be more acceptable. Furthermore, it was discovered that large problems like this one (10 phases with 10 states, 2-3 controls and numerous path constraints, endpoint constraints, etc.) require a large number of grid points or collocation points in the optimal solution which can lead to the slow processing of optimal solutions.

Another challenge for the optimal solution was the complexity of the thrust and mass flow rate tables for the booster; because the function was not smooth with time it required a high collocation point density and thus a higher-order Lagrange polynomial to accurately reflect the steep changes of thrust and mass flow rate of the booster. The use of a lower number of collocation points would result in a mis-interpretation of the table-data since the steep changes would not be accurately captured. A solution to this problem may be the substitution of a different function for the table data; for example Bryson ${ }^{18}$ uses hyperbolic trigonometric functions to model near-step discontinuities in the transonic region for aircraft optimal control problems.

\section{Conclusion}

$\mathcal{G P O P S}$ was well suited for solving this complex multi-stage highly constrained boost and hypersonic glide vehicle problem. Run time is vastly improved when table data can be fit to smooth functions. An optimal solution may be found with the proper implementation of equations of motion and variable constraints. Determining those bounds at times took finesse most likely due to using the internal auto-scaling function. Leaving the variable bounds too wide resulted in not finding the optimal solution. With the implementation of a user-defined auto scaling function or non-dimensionalization that issue may be resolved.

Future work related to this effort may involve HGV model changes to improve aerodynamic table fit, thus derivative smoothing. As mentioned earlier, user-defined scaling of the dynamic equations would eliminate the need for auto scaling and ensure that relative weighting of variables in the nonlinear problem solver is as equivalent as possible. New problems which can be solved with this simulation framework relate to range safety questions such as maximum glide range footprint and possibly minimization of risk to populated areas. In addition, the simulation will continue to be used to explore new hypersonic glide guidance methodologies and to evaluate their feasibility while minimizing different cost functions.

\section{References}

\footnotetext{
${ }^{1}$ Jorris, T. R. and Cobb, R. G., "Multiple Method 2-D Trajectory Optimization Satisfying Waypoints and No-Fly Zone Constraints," Journal of Guidance, Control, and Dynamics, Vol. 31, No. 3, May-June 2008, DOI: 10.2514/1.32354.

${ }^{2}$ Jorris, T. R. and Cobb, R. G., "Three-Dimensional Trajectory Optimization Satisfying Waypoint and No-Fly Zone Constraints," Journal of Guidance, Control, and Dynamics, Vol. 32, No. 2, 2009, pp. 551-572.

${ }^{3}$ Jorris, T. R., Common Aero Vehicle Autonomous Reentry Trajectory Optimization Satisfying Waypoint and No-Fly Zone Constraints, Ph.D. thesis, Air Force Institute of Technology, September 2007.

${ }^{4}$ Jorris, T. R., Schulz, C., Friedl, F., and Rao, A. V., "Constrained Trajectory Optimization Using Pseudospectral Methods," AIAA Atmospheric Flight Mechanics Conference and Exhibit, 18-21 August 2008, AIAA 2008-6218.

${ }^{5}$ Jorris, T. R., Schulz, C. S., and Rexius, S. R., "Improvements in pseudospectral based Hypersonic Vehicle Trajectory Generation via Enhanced Aerothermal Prediction Methods," 19th AAS/AIAA Spaceflight Mechanics Meeting, 9-12 February 2009.

${ }^{6}$ Carr, R. W., Jorris, T. R., and Downey, J. J., "Trajectory Analysis Program for Determining Range Safety Considerations using Multiple-Phase pseudospectral Optimization," 28th Aerodynamic Measurement Technology, Ground Testing, and Flight Testing Conference, edited by AIAA, 25-28 June 2012.

${ }^{7}$ Rao, A. V., Benson, D. A., Darby, C., Patterson, M. A., Francolin, C., Sanders, I., and Huntington, G. T., "Algorithm 902: GPOPS, A MATLAB Software for Solving Multiple-Phase Optimal Control Problems Using the Gauss Pseudospectral Method," ACM Transactions on Mathematical Software, Vol. 37, No. 2, April 2010, pp. 22.1-22.39, Article 22.

${ }^{8}$ Benson, D. A., Huntington, G. T., Thorvaldsen, T. P., and Rao, A. V., "Direct Trajectory Optimization and Costate Estimation via an Orthogonal Collocation Method," Journal of Guidance, Control, and Dynamics, Vol. 29, No. 6, NovemberDecember 2006, pp. 1435-1440.

${ }^{9}$ Garg, D., Patterson, M. A., and Darby, C. L., "Direct Trajectory Optimization and Costate Estimation of Finite-Horizon and Infinite-Horizon Optimal Control Problems Using a Radau pseudospectral Method," Computational Optimization and Applications, Vol. 49, No. 2, June 2011, pp. 335-358.

${ }^{10}$ Garg, D., Patterson, M. A., and Hagar, W. W., "A Unified Framework for the Numerical Solution of Optimal Control Problems Using pseudospectral Methods," Automatica, Vol. 46, No. 11, November 2010, pp. 1843-1851.

${ }^{11}$ Garg, D., Hager, W. W., and Rao, A. V., "Pseudospectral Methods for Solving Infinite-Horizon Optimal Control Problems," Automatica, Vol. 47, No. 4, April 2011, pp. 829-837.
} 
${ }^{12}$ Waddell, E. T. and Jorris, T. R., "Filtering and Statistics of Flight Test Instrumentation Data," 39th Annual International Symposium, edited by SFTE, 17-21 November 2008.

${ }^{13}$ Kirsten, P. W., "Development of a Fuel-Saving Flight Control System for the Space Shuttle Based on Flight Experience," AIAA/AHS/ASEE Aircraft Design Systems and Operations Meeting, 14-16 October 1985.

${ }^{14}$ Seltzer, R. M. and Rhodeside, G. R., "Fundamentals and Methods of High Angle-of-Attack Flying Qualities Research," NADC-88020-60, 1988.

${ }^{15}$ Vinh, N. X., Optimal Trajectories in Atmospheric Flight, Elsevier, 1981.

${ }^{16}$ Champion, K. S. W., O'Sullivan, W. J., and Teweles, S. T., "U.S. Standard Atmosphere, Committee on Extension to the Standard Atmosphere (COSEA)," 1962.

${ }^{17}$ Chapman, D. R., "An Approximate Analytical Method for Studying Entry Into Planetary Atmospheres," Technical Note 4276, National Advisory Committee for Aeronautics, 1958.

${ }^{18}$ Bryson, Jr., A. E. and Ho, Y.-C., Applied Optimal Control, Taylor \& Francis, 1975. 